Weißhaupt, Mark; Schneider, Ralf; Griesel, Clemens; Pfrang, Agnes

\title{
Digitale Erfahrung? Über das Lernen zwischen Instruktion und
}

\section{(Ko-)Konstruktion}

Holub, Barbara [Hrsg.]; Himpsl-Gutermann, Klaus [Hrsg.]; Mittlböck, Katharina [Hrsg.]; Musilek-Hofer, Monika [Hrsg.]; Varelija-Gerber, Andrea [Hrsg.]; Grünberger, Nina [Hrsg.]: lern.medien.werk.statt. Hochschullernwerkstätten in der Digitalität. Bad Heilbrunn : Verlag Julius Klinkhardt 2021, S. 87-102. - (Lernen und Studieren in Lernwerkstätten)

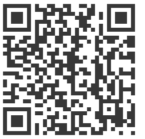

Quellenangabe/ Reference:

Weißhaupt, Mark; Schneider, Ralf; Griesel, Clemens; Pfrang, Agnes: Digitale Erfahrung? Über das Lernen zwischen Instruktion und (Ko-)Konstruktion - In: Holub, Barbara [Hrsg.]; Himpsl-Gutermann, Klaus [Hrsg.]; Mittlböck, Katharina [Hrsg.]; Musilek-Hofer, Monika [Hrsg.]; Varelija-Gerber, Andrea [Hrsg.]; Grünberger, Nina [Hrsg.]: lern.medien.werk.statt. Hochschullernwerkstätten in der Digitalität. Bad Heilbrunn : Verlag Julius Klinkhardt 2021, S. 87-102 - URN:

urn:nbn:de:0111-pedocs-228147 - DOI: 10.25656/01:22814

https://nbn-resolving.org/urn:nbn:de:0111-pedocs-228147

https://doi.org/10.25656/01:22814

in Kooperation mit / in cooperation with:

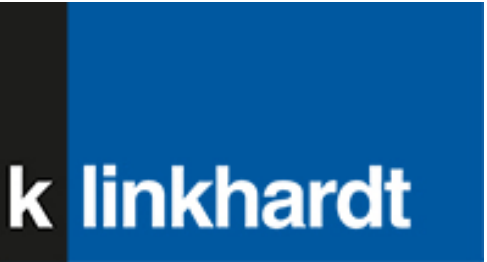

http://www.klinkhardt.de

\section{Nutzungsbedingungen}

Dieses Dokument steht unter folgender Creative Commons-Lizenz: http://creativecommons.org/licenses/by-nc-sa/4.0/deed.de - Sie dürfen das Werk bzw. den Inhalt unter folgenden Bedingungen vervielfältigen, verbreiten und öffentlich zugänglich machen sowie Abwandlungen und Bearbeitungen des Werkes bzw. Inhaltes anfertigen: Sie müssen den Namen des Autors/Rechteinhabers in der von ihm festgelegten Weise nennen. Dieses Werk bzw, der Inhalt darf nicht für kommerzielle Zwecke verwendet werden. Die neu entstandenen Werke bzw. Inhalte dürfen nur unter Verwendung von Lizenzbedingungen weitergegeben werden, die mit denen dieses Lizenzbedingungen weitergegeben werden,

Mit der Verwendung dieses Dokuments erkennen Sie die Nutzungsbedingungen an.

\section{Terms of use}

This document is published under following Creative Commons-License: http://creativecommons.org/licenses/by-nc-sa/4.0/deed.en - You may copy, distribute and transmit, adapt or exhibit the work in the public and alter, transform or change this work as long as you attribute the work in the manner specified by the author or licensor. You are not allowed to make commercial use of the work. If you alter, transform, or change this work in any way, you may distribute the resulting work only under this or a comparable license.

By using this particular document, you accept the above-stated conditions of

\section{Kontakt / Contact:}

\section{DeDOCS}

DIPF | Leibniz-Institut für Bildungsforschung und Bildungsinformation Informationszentrum (IZ) Bildung

E-Mail: pedocs@dipf.de

Internet: www.pedocs.de

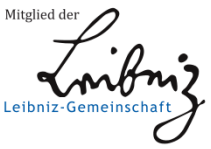




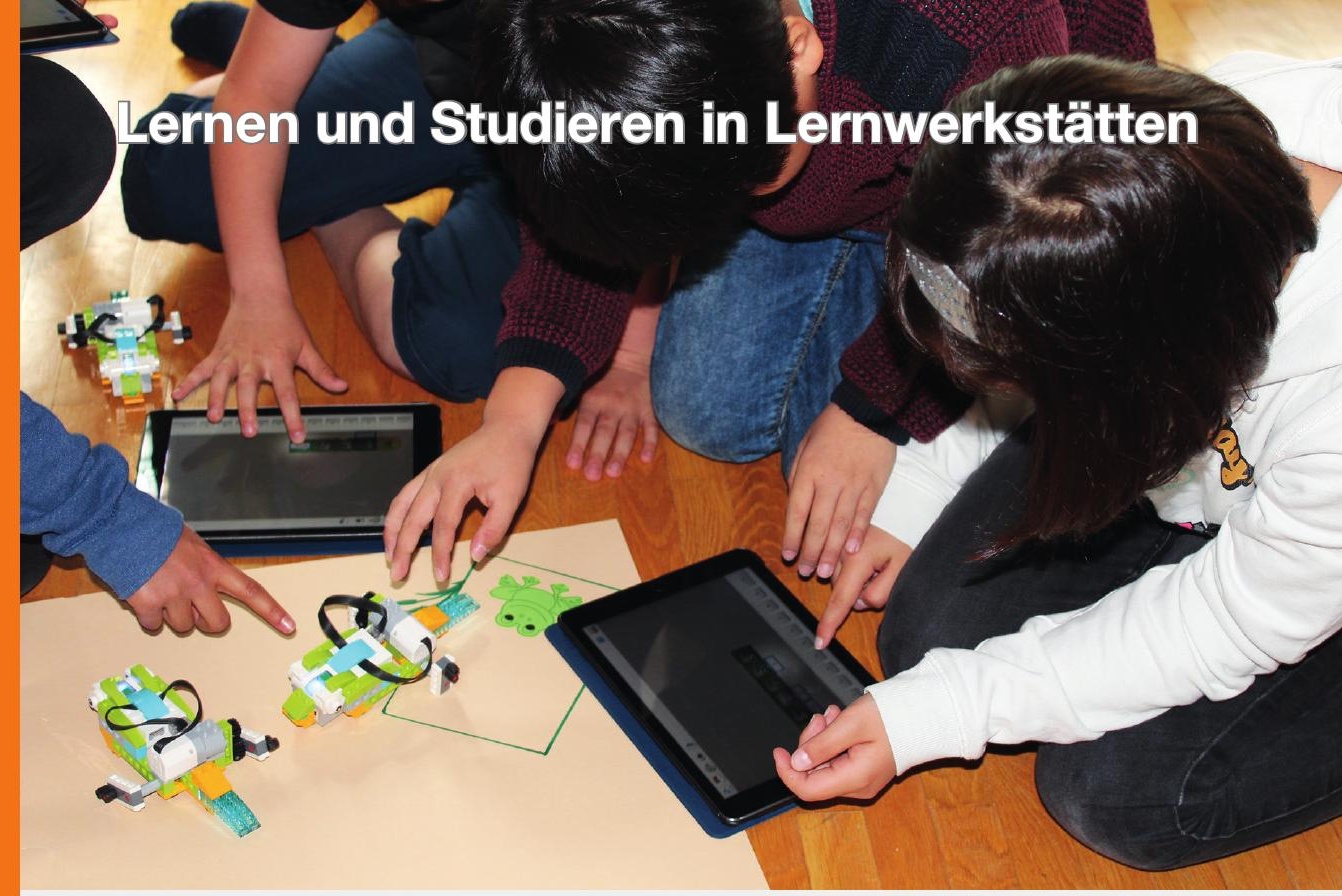

Barbara Holub / Klaus Himpsl-Gutermann Katharina Mittlböck / Monika Musilek-Hofer Andrea Varelija-Gerber / Nina Grünberger (Hrsg.)

\section{lern.medien.werk.statt}

Hochschullernwerkstätten in der Digitalität 
Barbara Holub Klaus Himpsl-Gutermann Katharina Mittlböck Monika Musilek-Hofer Andrea Varelija-Gerber

Nina Grünberger (Hrsg.)

\section{lern.medien.werk.statt}

Hochschullernwerkstätten in der Digitalität 
Dieser Titel wurde in das Programm des Verlages mittels eines Peer-Review-Verfahrens aufgenommen. Für weitere Informationen siehe www.klinkhardt.de.

Bibliografische Information der Deutschen Nationalbibliothek Die Deutsche Nationalbibliothek verzeichnet diese Publikation in der Deutschen Nationalbibliografie; detaillierte bibliografische Daten sind im Internet abrufbar über http://dnb.d-nb.de.

2021.ig. (C) by Julius Klinkhardt.

Coverfoto: (c) ZLI PH Wien.

Druck und Bindung: AZ Druck und Datentechnik, Kempten.

Printed in Germany 2021.

Gedruckt auf chlorfrei gebleichtem alterungsbeständigem Papier.

Die Publikation (mit Ausnahme aller Fotos, Grafiken und Abbildungen) ist veröffentlicht unter der Creative Commons-Lizenz: CC BY-NC-SA 4.0 International https://creativecommons.org/licenses/by-nc-sa/4.0/

ISBN 978-3-7815-5904-2 digital doi.org/10.35468/5904

ISBN 978-3-7815-2468-2 print 


\section{Inhaltsverzeichnis}

Barbara Holub, Klaus Himpsl-Gutermann, Katharina Mittlböck, Monika Musilek-Hofer, Andrea Varelija-Gerber und Nina Grünberger

Einführung in den Band 11

\section{Zur Konstituierung von Hochschullernwerkstätten}

Sandra Tänzer

Mut - Zumutung - Ermutigung. Oder wie man lernt, eine Hochschullernwerkstatt zu lieben

Markus Peschel, Hartmut Wedekind, Pascal Kihm und Mareike Kelkel

Hochschullernwerkstätten und Lernwerkstätten -

Verortung in didaktischen Diskursen

Dietlinde Rumpf und Corinna Schmude

Von der Herausforderung, die Vielfalt von Hochschullernwerkstätten

in einer Definition abzubilden

Pascal Kihm und Markus Peschel

„Komplexität wagen!“ - Methoden zur Beforschung von

offenen Lehr-Lern-Prozessen in Hochschullernwerkstätten 70

\section{Zum Spannungsverhältnis von Hochschullernwerkstätten und Digitalität}

Mark Weißhaupt, Ralf Schneider, Clemens Griesel und Agnes Pfrang

Digitale Erfahrung? Über das Lernen zwischen Instruktion und

(Ko-)Konstruktion

Stefan Brée, Lena S. Kaiser und Tanja Wittenberg

Lernwerkstatt als digitaler Erfahrungsort. Potenziale und

Herausforderungen für Lernwerkstätten als Orte

„offener multimedialer Produktionsästhetik“

\section{Ulrike Stadler-Altmann und Gerda Winkler}

Real \& virtuell, analog \& digital: Dimensionen einer Kooperation.

Multifunktionalität als Kennzeichen zukunftsfähiger Lernwerkstatt- und

Bibliothekskonzeption 


\section{Josef Buchner und Michael Kerres}

Lernwerkstattarbeit in der digital vernetzten Welt.

Die Perspektive der gestaltungsorientierten Mediendidaktik

Sabrina Tietjen und Silvia Thünemann

Forschungswerkstatt digital: ein modernes Lehr-Lernarrangement

für eine Digitalisierungsstrategie im Lehramt?

\section{Zu fach- und mediendidaktischen Perspektiven}

Johannes Mayer, Antonia Lemensieck, Maria Reinhardt und Karl Wollmann

Fachliche Perspektiven auf digitalisierungsbezogene Lernangebote

in der Ausbildung von Grundschullehrer*innen

Heike Hagelgans

Die Thematisierung digitaler Medien in den schulpraktischen Studien

Fachdidaktische Reflexionen von digitalen Medien für das Lernen im

Mathematikunterricht der Primarstufe

Jeanette Hoffmann, Katharina Egerer und Franziska Herrmann

Analoge Bilder - digitaler Film.

Möglichkeitsräume für literarästhetisches Lernen in

Hochschullernwerkstätten

Michael Rieseneder und Wolfgang Wagner

Erstes Programmieren mit Kindern über Handlungserfahrungen.

Das Konzept Activity-based-Coding

Patrick Isele und Julia Höke

Reflexionen über digitales Lernen mit dem Sphero SPRK+

Erfahrungen mit Studierenden in der „Lernwerkstatt ${ }^{3 “}$

Susanne Schumacher, Ulrike Stadler-Altmann und Enrico A. Emili

Piktogramme als Unterstützungsmedien.

Studien zu Effekten von Bilderbüchern mit zusätzlichen Symbolen im Kindergarten 


\section{Zu Aspekten, Perspektiven und Herausforderungen von Hochschullernwerkstätten}

Sabine Fischer und Max de Baey-Ernsten

Didaktische Perspektiven einer ästhetischen Werkstatt

Tanja Wittenberg und Lena S. Kaiser

„Ich war frustriert, ich bin kein Kind mehr“ - Erfahrungslernprozesse

mit verwendungs- und bedeutungsoffenem Material in

kindheitspädagogischen Hochschullernwerkstätten

Lisa Eßel und Laura Schlichting

Hochschullernwerkstätten im Spannungsfeld zwischen

Freiwilligkeit und Pflicht

Mareike Kelkel, Markus Peschel und Pascal Kihm

Potenziale der pädagogisch-didaktischen Öffnung in

Hochschullernwerkstätten

Barbara Holub und Sybille Roszner

Hochschullernwerkstatt - Ausgangspunkt für

persönlichkeitsorientierte Professionalisierung in der Ausbildung

für Lehrpersonen

Verzeichnis der Autor*innen 


\title{
Mark Weißhaupt, Ralf Schneider, Clemens Griesel und Agnes Pfrang
}

\section{Digitale Erfahrung?} Über das Lernen zwischen Instruktion und (Ko-)Konstruktion

\begin{abstract}
Es stellen sich angesichts eines forcierten Distance Learning und allgemein zunehmender Digitalisierung Fragen an den Stellenwert, die Bedeutung und den Umgang mit digitalen Medien: Welche Zusammenhänge bestehen zwischen kindlicher Welt-Erfahrung, Welt-Erkenntnis und digitalen Vermittlungsformen und -medien? Wie verändern sich sinnlich-leibliche Erfahrungen unter den Bedingungen von Digitalisierung? Was bedeutet das für das Lernen, speziell das entdeckende Lernen und das Lernen in Hochschullernwerkstätten?

Die Professionalisierung angehender Lehrer*innen muss sich hierbei einer inhärenten Beschleunigung stellen, da parallel zur technischen Entwicklung und deren marktförmiger Verbreitung eine kulturelle digitale Mediatisierung stattfindet. In unterschiedlichen Lernmilieus sind jedoch sehr ungleiche Kompetenzen und Praktiken mit digitalen Medien eingelebt, was zu folgenreichen Digital Divides führt-quer zur Unterscheidung Lehrende, Studierende, Kinder, was in Hochschullernwerkstätten Berücksichtigung finden muss.

In der Ausbildung von Lehrer*innen und in Hochschullernwerkstätten erscheint insofern keine Abkehr von digitalen Medien ratsam, sondern den Studierenden sind Lernumgebungen anzubieten, in denen sie erweiternd sowohl kritische Medienkompetenzen erwerben als auch leiblich-sinnliche Erfahrungen machen können, auch im Umgang mit technischen Geräten und Tools. Hieraus ergeben sich sowohl Widerstände und auch Lust am Medium, was durch Lehrende interaktiv genutzt werden sollte. Studierende sollten sich forschend und entdeckend den Bedingungen und Möglichkeiten des Lerngegenstandes und der digitalen Medien nähern, um Lernumgebungen für Kinder zu konstruieren, die sie auf entdeckendem Wege u. a. dazu befähigen, sich kritisch und reflexiv mit digitalen Medien auseinanderzusetzen. Alle Beteiligten in diesem Diskursraum, auch Lehrende, können hierbei von der Einnahme neuer Fragehaltungen profitieren.
\end{abstract}




\section{Erfahrungslernen und reale digitale Welt}

Eine der zentralen Fragen, die Arbeitende im pädagogischen Bereich und speziell in Lernwerkstätten beschäftigt (oder zumindest beschäftigen sollte), könnte man so formulieren: Wie, was, womit, wann, mit wem und wozu lernen Kinder? Für eine Pädagogik, die die aktive Auseinandersetzung von Kindern mit der Welt im sozialen Austausch als zentrales Element von Bildungsprozessen ansieht, stellen sich angesichts eines forcierten Distance Learning und allgemein zunehmender Digitalisierung insbesondere Fragen an den Stellenwert, die Bedeutung und den Umgang mit den digitalen Medien: Welche Zusammenhänge bestehen zwischen kindlicher Welt-Erfahrung, Welt-Erkenntnis und digitalen Vermittlungsformen und -medien? Was bedeutet das für das Lernen, speziell das entdeckende Lernen und das Lernen in Hochschullernwerkstätten?

\subsection{Digitales Lernen zwischen einer induktiv-kindlichen und einer deduktiv-erwachsenen Perspektive}

Nimmt man zunächst einmal die unterschiedlichen Typiken des Lernens Erwachsener und die Bedeutung des erfahrungsbasierten Lernens von Kindern sehr grob in den Blick und stellt sie idealtypisch einander gegenüber, so lassen sich wesentliche Unterschiede erkennen: Junge Kinder erfahren „Welt“, indem sie sie sich geradezu „einverleiben“ und auf einer sehr konkreten Ebene mit allen Sinnen ihres Körpers die Vielseitigkeit der Welt wahrnehmen und in all ihren Facetten im tätigen Gebrauchen immer weiter ausdifferenzieren (Meyer-Drawe, 2012). Im sozialen Austausch mit anderen werden dabei zunehmend kulturelle Denk-, Kommunikations- und Handlungswerkzeuge verwendet (Mead, 2005; Vygotski, 1969) und im Gebrauch zunehmend verfeinert.

Im Sinne eines kategorialen Bildungsverständnisses (Klafki, 1964) handelt es sich dabei um einen relationalen Prozess der Welt- und Icherkenntnis in Abhängigkeit von sozialen Austauschmöglichkeiten, in dem leiblich-sinnliche Erfahrung und kognitive Entwicklungen interferieren und sich - wenn auch nicht völlig bestimmbar - weiterentwickeln. In der Kindheit fußt dieser dynamische Entwicklungszusammenhang auf konkretes sinnliches Erfahren, erprobendes Handeln und entdeckendes Explorieren (Langeveld, 1968). Er sieht sich, nicht nur in institutionellen Vermittlungskontexten, einer erwachsenen Weltsicht gegenüber (nicht dichotom, sondern in einem Kontinuum gedacht), deren Erfahrungswelt eher im Bereich des Abstrakten zu verorten ist (was auch im Allgemeinen den Bildungszielen in Curricula entspricht) und in die Kinder erst langsam „hineinwachsen“. Die Erfahrungswelt Erwachsener hat sich gegenüber der kindlichen Erfahrungswelt entwicklungsbedingt weiter ausdifferenziert und spezifiziert, ist in vielerlei Hinsichten kokonstruktiv validiert und konstituiert. Erwachsene haben sich in den abstrakten, verallgemeinerten Ordnungen ihrer kulturellen Welt, d. h. 
in ihren Systematiken, Logiken, ihrer Geschichte, den Kategorien, Denktraditionen und Verfahren sowie in dem Habitus ihrer Milieus und Kulturen bereits zu einem gewissen, wenn auch unterschiedlichen Grad, eingerichtet. Sie müssen weniger induktiv lernen - sie können öfter, von bereits erlernten Abstraktionen und Schemata ausgehend, deduktiv ableitend auf den jeweils aktuellen Einzelfall schließen, und ihn so in vorhandene Schemata einordnen.

Eine Didaktik des entdeckenden Lernens bevorzugt - generell und auch in diesem Kontext -, dass Lernende möglichst selbstbestimmt Ziele, Wege und Methoden des Lernens finden und auf diesem Weg strategische Problemlösungsfähigkeiten erwerben, die sie dauerhaft auf neue (Erfahrungs-)Bereiche übertragen können (Schneider et al., 2019). Wer dem folgt, neigt zu Skepsis gegenüber einem Lernen, das zu schnell, zu kleinschrittig, zu einseitig und einförmig, oftmals zu früh, zu abstrakt, zu sprachlastig und zugleich erfahrungsarm organisiert wird. Diese Skepsis zielt auf eine zu erwachsenenorientierte Lehrperspektive ab, die kindliche Lernprozesse in klar vorgegebene Lernziele und Lernformen in von oben instruierten Ordnungsschemata organisiert (vgl. die Überlegungen zu Sprachbildungsprozessen bei Hummel \& Schneider, 2017).

Was hat dies alles nun mit den digitalen Medien zu tun? Wer dem Lernen mit digitalen Medien skeptisch bis ablehnend gegenübertritt, kann argumentieren, dass die konkrete sinnlich-leibliche Dimension der Erfahrung beim Lernen mit digitalen Medien auf bestimmte Weise in Frage gestellt bzw. tendenziell reduziert oder abgekürzt wird (Wiater, 2020), und dabei statt entdeckendem, konstruktivem Lernen - verstanden als tastendes und explorierendes Versuchen an der Welt -, eher das auch technisch bedingte vorschnelle Einordnen in gegebene abstrakte Ordnungen sowie nach Kriterien praktiziert wird, die selbst unbekannt sind und nicht miterschlossen werden.

Als ein illustrierendes Sachbeispiel folgt eine mögliche Praxis des Lernens in naturnahen Umgebungen: Was ist das für ein Blatt, was für eine Pflanze? Nimm die Bestimmungs-App, mach ein Foto und schon wird der lexikalische Eintrag der Pflanze dargestell, mitsamt Erscheinungsformen, Verbreitung, Gattungen etc.

Die Einordnung des Phänomens in eine Stelle der Kultur ist sofort und ohne Umwege direkt digital verfügbar. Was aus der Perspektive Erwachsener als ein hilfreiches und sofort nutzbares Instrument erscheinen kann, um neues mit altem Wissen zu ergänzen und zu vernetzen, indem Neues in vorhandene Informationsverarbeitungsstrukturen eingeordnet wird, stellt junge Nutzer*innen vor das Problem, weder bereits über diese ordnenden Strukturen zu verfügen, noch die Kriterien reflektieren zu können, die dieser Ordnung zugrunde liegen. Die App nimmt den Nutzer*innen die verarbeitende Fähigkeit ab, über die sinnliche Wahrnehmung die Pflanze selbst auf irgendeine Weise, sei es ästhetisch oder anderweitig, qualifizieren zu sollen bzw. sich dazu ausdrücken zu können. Bevor weitergehende Fragen auftauchen, scheint deren digital-synchrone und deduktive 
Beantwortung sie von vorneherein überflüssig zu machen. Ein induktives Vorgehen, ein individuell bedeutsamer Zugang zur Sache, eine eigene Fragestellung der Lernenden, die selbstgesteuertes und nachhaltiges Lernen unterstützen, scheinen in diesem Beispiel gar nicht erst entstehen zu können. ${ }^{1}$

In diesem Gedankenspiel kann man dieser extremen Auslegung jedoch auch andere Möglichkeiten gegenüberstellen, in denen explorative Tätigkeiten durch eine Änderung der Fragehaltung entstehen (können). Eine Gruppe älterer Kindergarten-Kinder bzw. jüngerer Schul-Kinder könnte sich z. B. zusammenfinden und mit einem zur Verfügung gestellten Digital-Tablet auf Exkursion begeben, um ihre „Lieblingsbäume“ in der realen Umgebung zu besuchen bzw. zu finden. Sie könnten sich vor Ort gegenseitig mit dem Tablet interviewen, was die jeweiligen Bäume für sie bedeutsam machen, was ihnen daran gefällt, was sie darüber wissen, noch nicht wissen und was sie darüber hinaus noch an Fragen dazu interessiert. In einem solchen Setting haben sie Raum, um zu staunen, Eindrücke der sinnlichen Wahrnehmungen auszutauschen, das Wahrgenommene zu beschreiben, zu vergleichen, zu messen, zu unterscheiden, vermutend zu ordnen und zu überprüfen. Anschließend könnte man Statements, Wissen und Fragen u. a. aus den Tonaufnahmen herausdestillieren und in der Gruppe gemeinsam überlegen, was sich daraus für Unterthemen ergeben. Dann könnte man individuellen und gemeinsamen Fragen nachgehen, eigene gemachte Fotos und Videos der Bäume gemeinsam untersuchen (auch mit Hilfe von Apps), deren Formen auf realem Papier oder in einer virtuellen Umgebung nachbilden, Bücher und andere Expert*innen einbeziehen, wieder gezielt losgehen, ... kurz: alle Möglichkeiten des entdeckenden Lernens nutzen (vgl. Kahls Film „Kinder“ (2008) und darin vor allem die Sequenz über Naturbeobachtungen). Am Ende wäre es möglich, Originalton-Ausschnitte und Bilder am Tablet zu einer Präsentation zusammenstellen, die den gemeinsamen und individuellen Weg des Projekts mitsamt Umwegen in Teilen dokumentiert, für die Einzelnen, für die Gruppe und ggf. für das Publikum der Eltern (wobei Datenschutzerwägungen hier natürlich noch eine Rolle spielen). Damit wäre ein Umgang mit digitalen Medien gewonnen, der Kindern die Möglichkeit gibt, nicht nur bruchstückhaft am Erwachsenenwissen teilzuhaben, sondern am eigenen Wissensaufbau über Weltausschnitte mit Hilfe zunächst selbst gewonnener Kriterien zu partizipieren.

Vor dem Hintergrund dieser Überlegungen zum Wissenssaufbau von Kindern, die alles andere als eine digital-pessimistischen Sicht widerspiegelt, kann man nun einwenden: Zwar scheint ein induktiver Zugang so auch mit digitalen Medien möglich, aber wieso sollte man dies so oder auf ähnliche Weise mit digitalen

1 In gewisser Hinsicht stellt die App hier eine Art interpassives Zwischenobjekt dar, das für den Benutzer/die Benutzerin die ,anstrengende Pflichtübung“ der Interaktion mit der Pflanze übernehmen soll (Pfaller, 2017; Weißhaupt \& Hildebrandt, 2020). 
Medien zu machen überhaupt erwägen, wenn es auch ganz ohne digitale Medien geht? Petko et al. (2018) haben (hier stellvertretend für viele andere) für die Lehrer*innenbildung gefordert, dass mediale Vorerfahrungen, mediale Themen und mediale Praktiken verstärkt im Studium behandelt und dabei auch erlebbar gemacht werden sollten. Neben der für uns interessanten Frage, was die Erlebnisdimension hier bedeutet, ist zu fragen: Warum eigentlich genau? Und: Hat dies nicht auch Rück- bzw. Kehrseiten, die es im Auge zu behalten gilt? Um das zu beantworten, soll ein kursorischer Umweg über die sich im Fluss befindliche Stellung der digitalen Medien in der Bildung und der Gesellschaft genommen werden.

\subsection{Digitale Verheißung und digitale Brüche in Bildung und Gesellschaft}

Der digitale Wandel wird von Petko et al. (2018, S. 160f.) so beschrieben:

Neben der technologischen hat der Wandel zudem eine gesellschaftliche und wirtschaftliche Seite, die u. a. mit Begriffen wie „Mediatisierung" (Krotz, 2015; Schulz, 2004) oder „Leitmedienwechsel“ (Honegger, 2016) beschrieben wird [...] eine grundlegende Veränderung in menschlichen Verhaltensweisen ergeben, die wiederum eng mit weiteren großen ... Trends zusammenhängen, etwa der Globalisierung, neuen Formen des Wettbewerbs oder der Nachhaltigkeit. (Dicken, 2014; Webster, 2014)

Neben berechtigten Nachfragen bzw. Einwänden, die man in Bezug auf eine hier postulierte „Nachhaltigkeit“ der Digitalisierung formulieren könnte (siehe z. B. Santarius, 2019), ist sicher richtig festzuhalten, dass die Lebenswelten, insbesondere auch die von Kindern, verstärkt von digitalen Medien mit strukturiert sind und dies zunehmend sein werden - unsere kulturellen Werkzeuge ändern sich diesbezüglich sehr schnell. Hierzu kann man im Wesentlichen zwei gegensätzliche Haltungen im Diskurs feststellen.

Auf der einen Seite wird auf das konstruktive Potenzial der digitalen Medien für die Bildung hingewiesen. Diese lägen nämlich nicht nur in einem möglichen Ersatz der Funktionen von bereits vorhandenen Medien. Wer die digitalen Medien wie die vor-digitalen benutze, habe deren Eigenarten nicht ausreichend wahrgenommen (Petko, 2019). Gerade die Möglichkeit, Lernen neu stärker zu personalisieren, d. h. zu individualisieren, statt alle in der Lerngruppe gleichförmig zu instruieren ${ }^{2}$, wird so als deren wichtiges Potenzial herausgestellt (ebda.).

2 Dies wird im Diskurs des Digitalen zuweilen als etwas dargestellt, was vor allem mit den neuen digitalen Möglichkeiten machbar sei (vgl. Petko, 2019), wodurch zentrale Ideen und Praxen reformpädagogischer Ansätze, Überlegungen zur Gestaltung entdeckender und problemorientierter Lernprozesse, die Diskussion um Öffnung von Unterricht unter lernpartizipativer Perspektive, den Sinn einer Laboratory School (Dewey), motivationstheoretische Überlegungen (Deci \& Ryan) und vieles mehr unsachgemäß ausgeblendet werden. Dennoch muss man sich mit dem Potenzial der digitalen Ko-Konstruktion inhaltlich auseinandersetzen. 


\section{Mark Weißhaupt, Ralf Schneider, Clemens Griesel und Agnes Pfrang}

Weiter werden als positive Potenziale digitaler Medien aufgeführt, Lernaufgaben könnten besser auf individuelle Erfordernisse und Schwierigkeitsstufen zugeschnitten werden, Lernprozesse könnten leichter gemeinsam in der Gruppe bearbeitet werden, Ergebnisse (unabhängig von lokaler Präsenz) geteilt werden, bei Schwierigkeiten schneller Hilfen abgefragt und individuelles Feedback erzeugt werden. Gerade eine neue ko-konstruktive und interaktive „Schwarm“-Kultur der Lernunterstützung werde durch den digitalen Wandel also erst qualitativ neu möglich und gelebt. Ein Modell der Transformation der Mittel und Ziele der Bildung statt nur der Modifikation oder bloßer Ergänzung sei in der Bildung zu vollziehen, bzw. es sei nachzuvollziehen, was in der Gesellschaft sich ohnehin in dieser Richtung digital wandele.

Nicht nur zu ihrem Vorteil, wird auf der anderen Seite argumentiert, denn neben einer möglichen, oben schon exemplifizierten Gefahr der Erfahrungsreduktion geht mit der Digitalisierung auch ein Trend zur permanenten Gleichzeitigkeit einher: Exemplarisch ist das Smartphone ein Tool, das örtliche Zeitlichkeit und lokal stattfindende, sachliche wie soziale Interaktion und Lernen statt zu verstärken auch sehr effizient unterbrechen kann - man denke an Push-Nachrichten, ablenkende Töne und die sozial um sich greifende Erfordernis instant auf Nachrichten und zu likende Mini-Häppchen der neuen Kommunikationskultur in den sogenannten sozialen Netzwerken zu reagieren. Das Smartphone ist zugleich das Tool bzw. die im Massenmarkt verbreitete Ware, die für die Welterfahrung von Kindern und Jugendlichen zunehmend bedeutsam wird. So werden tendenziell immer früher in der Kindheit auch Smartphones (nicht nur Tablets) von Kindern benutzt (bitcom Studie, 2019).

Zur Vergleichzeitigung kommt die Gefahr der Vereinheitlichung - auch in der Schule: Verlage entwickeln und implementieren fachliche Lernapps, die das soziale und didaktische Verhältnis zwischen Schüler*innen und Lehrer*innen in Zeiten des forcierten Distance Learning de facto zu ersetzen drohen, wenn die Lehrer*innen sich zusehends zurückziehen. Gerade das Gegenteil einer individualisierten Lernkultur, sondern eine Vereinheitlichung und Verarmung wirklich interaktiver, lokaler (Lern-)Formen werde durch digitale Medien unterstützt, so also das wesentliche Argument der Skeptiker*innen.

Wie schon Petko et al. (2018, S. 158) formulierten, stützen sich die „Argumente von euphorisierten Digitalisierungsbefürworterinnen und Digitalisierungsbefürwortern und alarmistischen Digitalisierungsgegnerinnen und Digitalisierungsgegnern oft nur auf einseitige Betrachtungsweisen, die nur einen Teilbereich des Digitalisierungstrends abdecken. " Der wesentliche Fehler, den einseitige Betrachtungen hierbei jeweils begehen, besteht u. E. darin, die digitalen Praktiken, losgelöst von ihren sozialen Bezügen, vor allem auf die Technik zu beziehen. Entscheidend sind aber die ungleiche Verteilung von Erfahrungen und produktiven Praktiken des Digitalen in der Gesellschaft, die Digital Divides. Denn sowohl unter 
Kindern und Studierenden als auch unter Lehrer*innen und Dozierenden sind die Erfahrungen und Kompetenzen im Umgang mit den digitalen Medien sehr unterschiedlich ausgeprägt, und das gilt für alle klassischen Baacke'schen Medienkompetenzen in den Bereichen Medienkritik, Medienkunde, Mediennutzung und Mediengestaltung.

Was ist ein Word-Dateiformat? Wann darf ich jemanden fotografieren, und was darf und kann ich mit dem Foto machen? Wie gestalte ich ein Bookcreator-Dokument, um unsere Forschungen in der Grundschulgruppe anderen zugänglich zu machen? Wie befrage ich Onlinewissen? Welchen Online-Erscheinungsformen von Personen oder Quellen kann ich vertrauen? Was ist eine Autorin, ein Autor? Was ist Wahrheit und was eine wissenschaftliche Haltung zu einem vorläufigen Stand des Wissens? Was für eine Art Wissen wird in sogenannten sozialen Netzwerken erzeugt und wie?

Die mit diesen Beispielen verknüpften Kompetenzen sind innerhalb aller genannten Gruppen von Partizipierenden an der digitalen Lernkultur sehr unterschiedlich verteilt. Auch die Rede von den „Digital Natives“ ist hier irreführend. Verschiedene Generationen haben zwar in prägungswirksamen Phasen ihrer Entwicklung sehr unterschiedliche zeitliche Erfahrungslagen (Mannheim, 1929) in Bezug auf bestimmte Medien, seit den 1980ern rasch zunehmend in Bezug auf digitale Medien (Heimcomputer, Internet-PC seit den 1990ern, Tablets und Smartphones seit den 2000er Jahren stark zunehmend). Auch sollte jedem im pädagogischen Feld Tätigen klar sein, dass generationale Medien auch immer mindestens teilweise Medien der Abgrenzung gegenüber den jeweils älteren Generationen sind und deshalb Versuche der „Förderung digitaler Kompetenzen“ von Jungen durch Ältere ggf. auf einen generationalen Peer-Widerstand stoßen können. Dennoch ist es ein nach wie vor weit verbreitetes grobes Missverständnis, dass Kinder gute Medienkompetenzen heute quasi von alleine, nebenbei ausbilden, denn das gilt eben nicht quer für alle Gleichaltrigen in der Gesellschaft (vgl. auch Spannagel, 2014).

Es gilt vielmehr unterschiedliche familiäre Hintergründe, d. h. Mediennutzungsmilieus in den Blick zu nehmen (Leopold \& Ullmann, 2018, S. 19). Digitale Praktiken werden mit den jeweils vorhandenen Ressourcen und dem verorteten Habitus selbstverständlich vor- und eingelebt. Hier entscheidet sich vor allem, wie sich digitale Medien und Bildungsinteressen zueinander verhalten. Nicht nur Unterschiede im schlicht quantitativen Zugang zu digitalen Medien (first order digital divides [Digital Divides erster Ordnung]), sondern qualitative Praktiken der aktiven gemeinsamen Rezeption und Verarbeitung zuhause oder eben auch der Vernachlässigung, des unterschiedlichen Geschmacks, der Auswahl und des Anspruchs bzw. der Verfeinerung der Interaktion in und mit den digitalen Medien, und der Aufmerksamkeit für diese im Verhältnis zu anderen Bereichen des Lebens, sind für dauerhaft starke Ungleichheiten und eingeschränkte bzw. 


\section{Mark Weißhaupt, Ralf Schneider, Clemens Griesel und Agnes Pfrang}

gestärkte Bildungsmöglichkeiten entscheidend (second und third order digital divides [Digital Divides zweiter und dritter Ordnung], Scheerder et al., 2017). ${ }^{3}$

\section{Strategien der Konstruktion entwickeln}

Wie stellt sich nun die Pädagogik des Entdeckenden Lernens der beschriebenen komplexen Problemlage? Im Folgenden sollen versuchsweise Strategien unterschieden bzw. entwickelt werden, um das Entdeckende Lernen und Möglichkeiten des Lernens an Hochschullernwerkstätten in diesem realen digitalen Kontext zu konturieren. Das Ziel dabei ist, nicht in einer „Kompensation“ bzw. „Komplementierung", also einem Ausgleich der möglichen Nachteile von digitalem Lernen als Ziel der Hochschullernwerkstatt zu enden (Wiater, 2020), sondern darüber hinaus - gerade auch angesichts der Fallstricke - produktive Strategien mit digitalen Medien aufzuzeigen. Dabei gehen wir von zwei sich in der Abfolge und Logik ergänzenden Leitfragen aus:

1. Kann die Lust ${ }^{4}$ am medialen Zugang zur Welt, am Gerät und Tool selbst für entdeckende (kindzentrierte) bzw. forschende (studierendenzentrierte) Lernprozesse erfasst und nutzbar gemacht werden?

Um diese Frage beantworten zu können, erscheint es bedeutsam, einen differenzierten Blick auf unterschiedliche mediale Angebote und Zugänge zu richten. Lernen als Erfahrung zu verstehen, verschließt sich auf den ersten Blick der Möglichkeit, die Lust am medialen Zugang zur Welt, am Gerät oder Tool selbst für entdeckende und forschende Lernprozesse fruchtbar machen zu können. Bei näherer Betrachtung kann das aber nur für mediale Praktiken gelten, die auf vorwiegend kognitivem bzw. instruktionalem Weg Fakten und Erkenntnisse vermitteln und Antworten als einzig richtige Lösungen präsentieren.

Beispielsweise kann auf der Lern-App „Anton“ Wissen über Ritter und Burgen erworben werden, indem Schüler*innen Informationen (teils) bildunterstützt lesen oder anhören können. Zum Abschluss gibt es einen Test, bei dem Antworten nur als entweder wahr oder falsch bewertet werden können. Eine Grenze solchermaßen gestalteter Lern-Apps für entdeckendes Lernen von Schüler*innen besteht darin, dass diese ihr Wissen lesend oder hörend erweitern können, leib-

3 Die 2020 in der Not erfolgte Umstellung auf Distance Learning für alle Schüler*innen ist deshalb in Sachen digitaler Pädagogik besonders kritisch zu sehen. Sehr ungleiche digitale Erfahrung, Engagement und Möglichkeiten bei den Lehrpersonen multiplizierte sich mit sehr ungleichen Möglichkeiten in den Familien.

4 Wir verwenden an dieser Stelle bewusst den Begriff „Lust“ und nicht „Interesse“, weil er unterhalb der Schwelle des Reflexiven liegt und nicht immer kognitiv operationalisierbar bzw. zugänglich ist, weder für die Akteur*innen, noch für die Beobachter*innen der Akteur*innen. 
lich-sinnliche Erfahrungen außerhalb des Bildschirms aber keinerlei Berücksichtigung finden. Auch der beim Lernen so wichtige Scaffolding-Prozess (Brush \& Saye, 2002) bleibt weitgehend unberücksichtigt. Zusätzliche Impulse, Herausforderungen oder Unterstützungsangebote, die bzw. eine richtige Lösung eigenständig zu finden, werden nicht integriert. ${ }^{5}$ Aus diesen kritischen Anmerkungen kann aber nicht per se gefolgert werden, dass die oft beobachtbare Lust von Kindern beim Umgang mit digitalen Medien, technischen Geräten und Tools nicht für entdeckende Lernprozesse genutzt werden kann. Vielmehr führt dies zu der Frage, welche medialen Zugänge, technischen Geräte und Tools dies zulassen, und wie diese von Lehrer*innen dahingehend eingesetzt werden könnten.

Ausschlaggebend ist hier die Annahme, dass sich Lernen nie ausschließlich kognitiv, sondern immer auch leiblich vollzieht. Leibliche Erfahrungsprozesse müssen somit auch beim Lernen mit technischen Geräten und Tools berücksichtigt werden, was einen reflektierten Einsatz erfordert.

An einem Beispiel soll die Dimension des Konnex zwischen sinnlicher Erfahrung und Medium veranschaulicht werden: Mit einer Mikroskop-Kamera, die mit einem PC verbunden ist, können Einblicke in einen Mikrokosmos ermöglicht werden, um anschaulich sichtbar zu machen, wie Tinte in unterschiedlich fein hergestelltes Papier (Briefpapier, Zeitungspapier, Löschpapier) eindringt und auf Grund der Feinheit der Struktur sich gänzlich anders im Papier verteilt (je feiner das Papier, desto feiner die Linien). Ersichtlich wird beim Lernen mit dieser Geräteverbindung, dass die Schüler*innen ihre Erkenntnisse selbst, handelnd und wahrnehmend, mit den Sinnen und mit dem Medium erweitern können, hierfür Durchhaltevermögen benötigen und zum Weiterdenken bzw. -lernen angeregt werden. Durch die Bereitstellung von Materialen oder thematischen Anreizen können Lehrende die Lernenden unterstützen und (neue) Impulse setzen. Die Antwort auf die Ausgangsfrage ist folglich auch von der Auswahl und dem Einsatz von Geräten und Tools abhängig.

Für die Professionalisierung angehender Lehrer*innen in Hochschullernwerkstätten ergibt sich in Konsequenz die Notwendigkeit, für Studierende Lernsettings zu schaffen, in denen sie den leiblichen Zugang zu Geräten und Tools selbst erfah-

5 Man kann an dieser Stelle berechtigterweise fragen, ob man bei einem Sachbuch zum Thema „Ritter" statt einer App in gleicher Weise kritische Fragen zu stellen bzw. Ansprüche zu formulieren geneigt wäre. Dies verweist auf eine allgemeine Anforderung an multimodale Lernumgebungen, inkl. verschiedener Medien und - nicht zuletzt - leiblich erfahrbarer Elemente, im Beispiel neben dem Sachbuch je nach Alter der Kinder ggf. ein Ausflug in ein Burgmuseum mit realen interaktiven Erfahrungselementen, eine aktive Auseinandersetzung mit Minnekultur, die Thematisierung, Infragestellung und Erweiterung von popkulturellen Bildern des Rittertums, evtl. zusätzlich die Erfahrung und Auseinandersetzung mit einem Videogame mit dem Thema der Simulation des Ritterlebens. 
ren, u. a. um später als Lehrende gezielt solche auswählen zu können, die dem sinnlich-leiblichen Lernen ihrer Schüler*innen Raum geben.

Forschendes Lernen in Hochschullernwerkstätten muss dabei dem Anspruch gerecht werden, kein Faktenwissen einzutrichtern, sondern vielmehr das Verstehen und Durchdringen von Begriffen, Phänomenen und Prozessen zu unterstützen. Um die Aufmerksamkeit der Studierenden durch Geräte und Tools zu wecken, müssen diese die Lernenden betreffen, sich bemerkbar machen bzw. sie affizieren (Heidegger, 1977, S. 86), was diese auch abhängig von der je eigenen familiärbiographischen Vorerfahrung in unterschiedlicher Art und Weise tun (ablenkend, dienend, kognitiv einschränkend oder anregend, ästhetisch verarmend oder aber erweiternd). So verstanden bieten digitale Medien, technische Geräte und Tools sich auch als Erfahrungsmöglichkeiten per se an. Aber nicht nur durch die reine Anwesenheit derselben reagieren die Studierenden, „sondern weil sie von sich aus Ansprüche haben, Herausforderungen bedeuten und Appelle aussenden. [...] Sie appellieren an mich, etwas zu tun oder zu unterlassen" (Meyer-Drawe, 2012, S. 182). Damit das Gemüt der Studierenden berührt, aber auch im Lernprozess habituell erweitert werden kann, muss ihm ein - vielfältiger, nicht zuletzt hochschuldidaktischer - Anspruch entgegentreten, den sie sich nicht selbst setzen, sondern auf den sie vielmehr antworten. In Bezug auf das Lernen lässt sich folgern, dass die Erfahrung der Studierenden durch entgegentretende Widerstände produktiv irritiert wird, die in Auseinandersetzung mit den digitalen Medien, technischen Geräten und Tools, und durch eine eingeforderte didaktische und reflexive Durchdringung derselben entstehen können. Für die Lehrenden ergibt sich daraus die Konsequenz, dass sie erstens bei den Studierenden Lernprozesse anstoßen bzw. in Bewegung setzen können, indem sie vielseitige Gelegenheiten bzw. Möglichkeiten schaffen, die den Erfahrungen der Lernenden Widerstand bieten, und dass zweitens neben dem Wissen über digitale Medien, technische Geräte und Tools eine praktizierte forschende Lernhaltung umso nötiger wird, in welcher konkret Wissen um kindliche Erfahrungsdimensionen beim Umgang mit diesen Geräten und Tools mit aufgebaut, reflektiert und aktiv gehandhabt werden muss.

2. Wie kann die doppelte Adressierung digitaler Medien an Kinder reflexiv für entdeckende Lernprozesse und Studierende in Lernwerkstätten an Hochschulen eingesetzt werden?

Im Zusammenhang mit dem Lernen in Hochschullernwerkstätten ist unter einer „zweifachen Adressierung“, im Gegensatz zu der verkürzenden und z. T. auf Seiten der Studierenden mit Gefühlen der Infantilisierung verbundenen Verständnis eines „didaktischen Doppeldeckers“, ein sehr viel tiefergehendes und voraussetzungsvolles Lehr-/Lernverständnis intendiert. Bei der zweifachen Adressierung geht es um die Suche nach Lerngegenständen, die zum einen Studierende her- 
ausfordern, sich forschend-lernend damit auseinanderzusetzen, als auch darauf aufbauend sie befähigen, Lernumgebungen für Kinder zu den gleichen Lerngegenständen zu entwickeln, in denen sie eine situativ, kontextbezogen, motivational, kommunikativ und auf die Lernentwicklung des Kindes angepasste dingliche, Wissens- und Herausforderungsstruktur generieren und mit didaktischem, vernetzendem, fachlich und fachdidaktischen Wissen in Verbindung bringen (Schneider et al., 2019, S. 98; Schneider, 2016).

Ein medienspezifisch kritisches Instrumentarium zu entwickeln, ist hierfür, neben den oben hervorgehobenen erfahrungsbezogenen und didaktischen Dimensionen, ebenfalls entscheidend. Im Hinblick auf das Lernen mit digitalen Medien besteht nach dem KMK-Strategiepapier zur Bildung in der digitalen Welt der Bildungsauftrag der Hochschulen darin, Studierende auf den Einsatz, den Umgang und hinsichtlich einer kritischen Reflexion digitaler Medien vorzubereiten (KMKStrategie, 2016, S. 42). Die dort formulierten Kompetenzen lassen sich durchaus mit dem Lernen in Hochschullernwerkstätten zusammenführen (ebda., S. 10f.). Das selbständige und nach eigenen Interessen geleitete Suchen, Verarbeiten und Aufbewahren stellt die Operationen dar, die in einem induktiven Prozess die Transformation von konkreten Erfahrungen zu abstrakten Begrifflichkeiten bestimmen. Darüber hinaus gilt es, die erworbenen (Er-)Kenntnisse zu kommunizieren und sie kooperativ weiterzuentwickeln. Weitere Ziele dabei sind das schrittweise Erwerben von Problemlöse-, Handlungs- und Reflexionskompetenz: Diese reichen von basalen Fähigkeiten wie dem Ausrichten eines Beamers bis hin zur Erstellung einer bedarfsgerechten, didaktisch reflektierten Powerpoint-Präsentation.

Diese hochschuldidaktischen wie didaktischen Fragen des Erwerbs und der Vermittlung digitaler Medienkompetenz steht einer sich immer weiter entwickelnden technisch-kommunikativen Umwelt und einer Vielzahl unterschiedlicher Akteur*innen und Interessen gegenüber, die sich hinter den technischen Geräten und Applikationen verbergen, welche selbst erst einmal durchdrungen werden will. Es geht schon lange nicht mehr um die „analogen Fragen“ nach einer hohen Druckauflage von bspw. enzyklopädischen Printmedien. Vielmehr rücken zunehmend die Nutzer*innendaten und ihr Nutzungsverhalten in den Mittelpunkt. U. a. bei kostenfreien Apps ist das Kunden-Produkt-Verhältnis hinsichtlich steuernder Abhängigkeiten zu be- und hinterfragen. Digitale Medien sind, wie im Prinzip jedes Medium, nicht per se reflexiv, sondern in erster Linie funktional und derzeit nicht zuletzt auf pekuniären Erfolg im Markt ausgelegt. Der Erwerb einer kritisch-reflexiven Grundhaltung ist also nicht im Produkt selbst zu finden, sondern obliegt den Nutzer*innen und der der Technik stets hinterherlaufenden gesellschaftlichen Reflexion, und ist im Bildungsprozess von hoher Bedeutung, was sich in der praktischen Medienarbeit, auch der digitalen, der Hochschullernwerkstatt zeigen sollte. 
Im Hinblick auf eine kritische Betrachtung digitaler Medien führt Wiater (2020, S. 140) Desozialisierung, Fremdsteuerung, eingeschränkte Realitätswahrnehmung und die fehlende Körpererfahrung als die zentralen Gefahren digitalen Lernens an, die sich aber hinsichtlich ihrer Operationalisierung für Lernprozesse etwas sperrig zeigen. Kombiniert man diese aber mit den Qualitätsmerkmalen für die Konstruktion von Lernumgebungen, wie sie Hummel und Schneider (2017) vorgelegt haben, lassen sich Übertragungsmöglichkeiten auch auf die Entwicklung von Lernumgebungen mit digitalen Medien finden, sofern sie Potenzial für medienkritische und zugleich entdeckende Lernprozesse bereitstellen. Wie bedeutsam dieser Fokus für entdeckendes Lernen sein kann, soll folgendes Beispiel verdeutlichen:

Für die digitale Verarbeitung von Bildern zu einem Film können Kinder zunächst mit Hilfe einer App zuvor gemalte Bilder, die sich in Details und sichtbaren Veränderungen (Bewegung von Gegenständen im Bildraum) zum vorherigen Bild unterscheiden, durch Einscannen dieser Bilder zu einem Film verarbeiten. So „lernen“ nach einem semi-technischen Verfahren „Bilder das Laufen“, indem ein Film, ein ansehnliches Produkt, entsteht. Nähert man sich in dieser technischen ausgerichteten Weise dem Thema an, werden viele Erfahrungsmöglichkeiten für Kinder und im Sinne einer zweifachen Adressierung auch für Studierende zu schnell übergangen. Mit Hilfe einfacher Sinnesübungen, z. B. dem Aufstellen und Aneinanderreihen von Standbildern, wie auch in der Auseinandersetzung mit Daumenkinos könnten Sinnhorizonte entdeckt werden, die Vermutungen darüber zulassen, wie das Auge im Zusammenspiel mit dem Gehirn und den erlernten Sehgewohnheiten im raschen Wechsel von Bildfolgen selbstständig einen Film erzeugen kann. Dieses Wissen verrät der Umgang mit Bildern in der App nicht. Hier muss man Kindern (ggf. abstrakt sprachlich) vermitteln, was sie selber durch kleine Versuche hätten entdecken können. Kinder laufen ohne breite Vergleichsperspektiven anhand zusätzlicher Objekte und Vergleichsmöglichkeiten, wie hier z. B. das Daumenkino, Gefahr, die Handlungsweisen für die technisch-digitale Bildverarbeitung zu übernehmen, ohne diese zu reflektieren oder zu hinterfragen. Die App allein provoziert Handlungen, fordert jedoch keine aktive Auseinandersetzung heraus (vgl. hierzu Kleickmann, 2012).

Studierende (und auch Dozierende und Kinder) haben wie bereits erwähnt sehr unterschiedliche Vorerfahrungen im Umgang mit digitalen Medien, die sich jedoch zu oft auf eine sichere Beherrschung bzw. Anwendung des technisch gegebenen Mediums beziehen, nicht aber auf eine medienkritische und -kundige Vergleichsperspektive oder eine sachbezogen-didaktische Durchdringung. Die Beherrschung des Mediums, der App oder des Gerätes ist kein Zeichen für eine reflexive Auseinandersetzung, sondern fördert in erster Linie eine Könnerschaft über eine informelle oder instruktionale Herangehensweise. Dies bestätigt auch eine Metaanalyse zum digitalen Lernen im Unterricht: Der Zuwachs von 
Lerneffektivität ist gegeben, fällt jedoch gering aus. Die Ergebnisse lassen darauf schließen, dass in einem schüler*innen- und problemorientierten Unterricht die Lerneffektivität höher ist als in darbietendem Unterricht. Dadurch ist die Art der didaktischen Einbettung möglicherweise auch eher ausschlaggebend (Schaumburg, 2018, S. 37) als die zukünftig noch genauer zu erforschenden - zunächst nur medial bedingten - Lernpotenziale digitaler „Schwarm“-Ko-Konstruktionen. Diese werden aber - wie vermutet werden kann - in ihrer Effektivität nicht zuletzt ebenfalls von Erfahrungsdimensionen inner- und außerhalb des Mediums selbst abhängen, von Widerstand bietenden didaktischen Herausforderungen und von einem zunehmend kritischen Durchschauen der jeweiligen medialen Gesetzmäßigkeiten. Die (potenziell) medienerfahrenen und -durchschauenden Nutzer*innen werden auch die Mitkonstrukteur*innen von künftigen Zugängen zu den dann neuen Medien sein, und diese Kompetenzen brauchen.

\section{Fazit}

Im Hintergrund stand nicht zuletzt die Frage: Wie verändern sich sinnlich-leibliche Erfahrungen unter den Bedingungen von Digitalisierung und welche Folgen hat dies für das Selbstverständnis, die Interessen bzw. die Lust und die Lernprozesse der unterschiedlichen digitalen Akteur*innen? Die Professionalisierung angehender Lehrer*innen muss sich hierbei einer inhärenten Beschleunigung stellen, da parallel zur technischen Entwicklung und der marktförmigen Verbreitung eine kulturelle digitale Mediatisierung stattfindet. Dies schafft sowohl zusätzliche Lern- und Erfahrungsmöglichkeiten als auch zusätzliche Verletzbarkeit von kindlichen wie erwachsenen Welten, und zwar in unterschiedlichen Lernmilieus, was zu komplexen und für das Lernen folgenreichen Digital Divides führt.

Kinder sind auf der Suche nach einem eigenen Verhältnis zur Digitalität und zur Umwelt. In der Auseinandersetzung mit (digitalen) Lerngegenständen sollen sich Studierende forschend den Bedingungen und Möglichkeiten des Lerngegenstandes nähern, um Lernumgebungen für Kinder zu konstruieren, die sie auf entdeckendem Wege dazu befähigen, sich kritisch und reflexiv mit digitalen Medien auseinanderzusetzen. Für eine thematische und inhaltliche Durchdringung ist keinesfalls eine Abkehr von Apps o. ä. gemeint, vielmehr dienen sie als Vehikel, Problemlöser und Helfer für das Durchdringen der Welt. Erst jedoch durch die sinnlichen und ganzheitlichen Entdeckungsprozesse werden die Grundlagen für eine reflexive Haltung geschaffen, die einem tautologischen Erklären entgegenwirken. In diesem Sinne gewinnen die Überlegungen zu einer zweifachen Adressierung Relevanz: 
Erst der Fokus auf das eigene entdeckende und forschende Lernen am (digitalen) Lerngegenstand bringt die Studierenden der Lernperspektive von Kindern näher, um dadurch die Besonderheiten digitaler Medien didaktisch zu durchdringen. In der Ausbildung von Lehrer*innen erscheint es der Argumentation folgend bedeutsam, den Studierenden Lernumgebungen anzubieten, in denen sie in reflektiertem Wechselverhältnis sowohl Medienkompetenzen erwerben als auch leiblich-sinnliche Erfahrungen im Umgang mit technischen Geräten und Tools machen können.

Unsere Überlegungen dienen als Impuls für die Gestaltung von Lernumgebungen mit digitalen Medien. Dabei zeigte sich die Kategorie des Widerständigen als bedeutsam für eine Erweiterung des je vorhandenen Reflexions- und Handlungshabitus, des Widerständigen sowohl innerhalb der Geräte und Medien und ihres Appells als auch der Widerstand in der (hochschul-)didaktischen Rahmung und der dortigen Interaktion. Alle Beteiligten in diesem Diskursraum, auch Lehrende, können dabei von der Einnahme neuer Fragehaltungen profitieren, die u. a. in Vergleichspraktiken in der Lernwerkstatt eingeübt werden können, wie sie in den obigen drei Beispielen aufschienen (Dokumentation, aber auch Verarbeitung der Baum-Exploration vor Ort mit dem Tablet, die sinnliche Dimension des GerätWahrnehmung-Konnexes beim PC-Mikroskop, Erfahrung und Reflexion des Verhältnisses Daumenkino/Einzelbildfilm-App). Die eigene Erfahrung ermöglicht es, den Weg der zweifachen Adressierung von (auch digitalen) Lernumgebungen zu durchlaufen, bis hin zur Frage: Wie kann ich mit Kindern die Vor- und Nachteile bestimmter Geräte bzw. nicht-medialer Zugänge zu den Dingen erfahrbar und damit der Reflexion zugänglich machen? Diesen komplexen Bereich nicht den Techniker*innen des Digitalen zu überlassen, sondern die qualitativen Digital Divides gemeinsam zu überwinden, muss eine Aufgabe sein, der sich auch Hochschullernwerkstätten widmen, gerade weil sie sich nicht zuletzt der Unterstützung des kindgerechten Lernens verschrieben haben.

\section{Literatur}

BITKOM (Bundesverband Informationswirtschaft, Telekommunikation und neue Medien e. V.) (2019). BITKOM-Studie 2019. Mit 10 Jahren haben die meisten Kinder ein eigenes Smartphone. https://www.bitkom.org/Presse/Presseinformation/Mit-10-Jahren-haben-die-meisten-Kinder-eineigenes-Smartphone

Brush, T. A., \& Saye, J. W. (2002). A Summary of Research Exploring Hard and Soft Scaffolding for Teachers and Students Using a Mulitmedia Supported Learning Environment. The Journal of Interactive Online Learning, 1(2), Fall 2002. http://www.ncolr.org/jiol/issues/pdf/1.2.3.pdf

Dicken, P. (2014). Global Shift: Mapping the Changing Contours of the World Economy (7. Aufl.). Sage Publications.

Heidegger, M. (1977): Phänomenologische Interpretation von Kants Kritik der reinen Vernunft. Ders.: Gesamtausgabe. II. Hrsg. von I. Görland. Abteilung: Vorlesungen 1923-1944. Band 25. Vittorio Klostermann.

Honegger, B. D. (2016). Mehr als 0 und 1: Schule in einer digitalisierten Welt (2. Aufl.). hep verlag. 
Hummel, M. \& Schneider, R. (2017): Offene Projektarbeit in der Lernwerkstatt als Ort für SprachBildung und Bildungs-Sprache. In M. Kekeritz, U. Graf, A. Brenne, M. Fiegert, E. Gläser, \& I. Kunze (Hg.), Lernwerkstattarbeit als Prinzip. Möglichkeiten für Lehre und Forschung (133-146). Klinkhardt.

Kahl, R. (2008). Kinder! - Reinhard Kahl über das Lerngenie der Kinder (24.04.2008). https://www. youtube.com/watch?v=mYD9JfOc26A

Klafki, W. (1964). Das pädagogische Problem des Elementaren und die Theorie der kategorialen Bildung (3./4., durchges. u. erg. Aufl.). Beltz.

Kleickmann, T. (2012). Kognitiv aktivieren und inhaltlich strukturieren im naturwissenschaftlichen Sachunterricht: Handreichungen des Programms SINUS an Grundschulen (IPN-Materialien) (IPN - Leibniz-Institut für die Pädagogik der Naturwissenschaften und Mathematik., Hg.). https:// pure.ipn.uni-kiel.de/portal/de/publications/kognitiv-aktivieren-und-inhaltlich-strukturieren-imnaturwissenschaftlichen-sachunterricht(7bfdeeda-605d-488c-a800-540cf3813327).html

KMK (Hg.) (2016). Bildung in der digitalen Welt. Strategie der Kultusministerkonferenz. https://www. kmk.org/fileadmin/Dateien/veroeffentlichungen_beschluesse/2018/Strategie_Bildung_in_der_ digitalen_Welt_idF._vom_07.12.2017.pdf

Krotz, F. (2015). Mediatisierung. In A. Hepp, F. Krotz, S. Lingenberg, \& J. Wimmer (Hg.), Handbuch Cultural Studies und Medienanalyse (439-452). Springer VS.

Langeveld, M. J. (1968). Studien zur Anthropologie des Kindes (2. durchges. und erg. Aufl. Reprint 2012). De Gruyter.

Leopold, M., \& Ullmann, M. (2018). Digitale Medien in der Kita: Alltagsintegrierte Medienbildung in der pädagogischen Praxis. Herder.

Mannheim, K. (1929). Das Problem der Generationen. Kölner Vierteljahreshefte für Soziologie 2/1928 (7).

Mead, G. H. (2005). Geist, Identität und Gesellschaft aus der Sicht des Sozialbehaviorismus: Aus der Sicht des Sozialbehaviorismus (14. Aufl.). Suhrkamp.

Meyer-Drawe, K. (2012). Diskurse des Lernens (durchges. und korr. 2012). Wilhelm Fink.

Petko, D. (2019). Personalisiertes Lernen mit digitalen Medien. Forum für Universität und Gesellschaft, Veranstaltungsreihe DIGITALE WELT - ANALOGE ERFAHRUNG (27.02.2019). https:// www.youtube.com/watch?v=v4veA6ZJ_9E

Petko, D., Honegger, B. D., \& Prasse, D. (2018). Digitale Transformation in Bildung und Schule: Facetten, Entwicklungslinien und Herausforderungen für die Lehrerinnen- und Lehrerbildung. Beiträge zur Lehrerinnen- und Lehrerbildung 36(2), 157-174.

Pfaller, R. (2017). Little Gestures of Disappearance: Interpassivity and the Theory of Ritual. In ders., Interpassivity - The Aesthetics of Delegated Enjoyment (53-68). Edinburgh University Press.

Santarius, T. (2019). Auf dem Weg in die vernetzte (Verbraucher-)Zukunft - Widersprüche der Digitalisierung für den nachhaltigen Konsum. In B. Bättel-Mink \& P. Kenning (Hg.), Paradoxien des Verbraucherverhaltens. Dokumentation der Jahreskonferenz 2017 des Netzwerks Verbraucherforschung (101-111).

Schaumburg, H. (2018). Empirische Befunde zur Wirksamkeit unterschiedlicher Konzepte des digital unterstützten Lernens. In N. McElvany, F. Schwabe, W. Bos, \& H. G. Holtappels (Hg.), Digitalisierung in der schulischen Bildung: Chancen und Herausforderungen (27-40). Waxmann.

Scheerder, A., Deursen, A. van, \& Dijk, J. van. (2017). Determinants of Internet skills, use and outcomes: A systematic review of the second- and third-level digital divide. Telematics and Informatics 34(8), 1607-1624. https://doi.org/10.1016/j.tele.2017.07.007

Schneider, R. (2016). Vom entdeckenden zum forschenden Lernen - eine hochschuldidaktische Herausforderung für Lernwerkstätten. In S. Schude, D. Bosse, \& J. Klusmeyer (Hg.), Studienwerkstätten in der Lehrerbildung: Theoriebasierte Praxislernorte an der Hochschule (107-118.). Springer VS. 


\section{Mark Weißhaupt, Ralf Schneider, Clemens Griesel und Agnes Pfrang}

Schneider, R., Weisshaupt, M., Brumm, L., Griesel, C., \& Klauenberg, L. (2019). Lernumgebungen in Hochschullernwerkstätten - Potenziale und Herausforderungen einer zweifachen Adressierung. In R. Baar, S. Trostmann, \& A. Feindt (Hg.), Struktur und Handlung in Lernwerkstätten. Hochschuldidaktische Räume zwischen Einschränkung und Ermöglichung (95-108). Klinkhardt.

Schulz, W. (2004). Reconstructing Mediatization as an Analytical Concept. European Journal of Communication 19(1). https://doi.org/10.1177/0267323104040696

Spannagel, C. (2014). „10 Irrtümer zum Einsatz digitaler Medien in der Schule“. Im Rahmen der Veranstaltung „Lernen zwischen Fibel und Youtube“ in der Berliner Stiftungswoche 2014. https:// cspannagel.wordpress.com/2014/12/15/10-irrtumer-zum-einsatz-digitaler-medien-in-der-schule/

Vygotskij, L. S. (1969). Thought and language. (4. Aufl.). The M.I.T. Press.

Webster, F. (2014). Theories of the Information Society (4. Aufl.). Routledge.

Weißhaupt, M., \& Hildebrandt, E. (2020). Delegiertes Spielen-Aufgeführtes Lernen. In U. StadlerAltmann, S. Schumacher, E. A. Emili, \& E. Dalla Torre (Hg.), Spielen, Lernen, Arbeiten in Lernwerkstätten. Facetten der Kooperation und Kollaboration (29-39). Klinkhardt.

Wiater, W. (2020). Lernwerkstätten in Zeiten des digitalen Lernens. In U. Stadler-Altmann, S. Schumacher, E. A. Emili, \& E. Dalla Torre (Hg.), Spielen, Lernen, Arbeiten in Lernwerkstätten. Facetten der Kooperation und Kollaboration (135-147). Klinkhardt. 